\title{
Disagreement loop and path creation/annihilation algorithms for binary planar Markov fields with applications to image segmentation
}

\author{
T. Schreiber, Nicolaus Copernicus University, Torun, Poland \\ M.N.M. van Lieshout, CWI/EURANDOM, The Netherlands
}

June 29,2007

\section{Introduction}

A classical problem in image analysis is that of segmenting the data into relatively homogeneous areas (see e.g. Rosenfeld \& Kak (1982)). It is often the first step in further analysis. Given the centrality of the problem, it is not surprising that a myriad of segmentation methods has been proposed, both deterministic and stochastic in nature. Indeed, the first seminal papers in statistical image analysis (Besag (1986), Geman \& Geman (1984)) concerned this problem. The authors proposed using Markov random fields - sometimes also referred to as Gibbs distributions or fields - to favour spatially coherent image partitions over more noisy ones. More precisely, a graph is formed by taking the pixel lattice as vertices, joining nearby pixels by an edge between them, and assigning high probability to images in which pixels sharing an edge have similar values. A more recent account of this approach can be found in the volume edited by Chellapa \& Jain (1993), or the textbooks by Gimel'farb (1999) and Winkler (2003).

The approach described above uses models that operate on the pixel level. Alternative methods focus on the partition of the image that is the outcome of a segmentation. Green (1995) and Møller \& Skare (2001) propose Voronoi based models, and Nicholls (1998) suggests triangulations. In previous work, Kluszczyński et al. (2005, 2007) advocated the use of polygonal field models (introduced in a range of papers by Arak, Clifford \& Surgailis $(1989,1991,1993))$, an idea entertained by Clifford \& Middleton (1989). In contrast to Markov random fields, the coloured Arak \& Surgailis fields take as starting 
point not the pixel lattice but the Poisson line process. Any realisation of this process induces a tessellation, which is then coloured. The boundaries between different-coloured regions form the edges of a graph. Arak \& Surgailis (1989) showed that a careful choice of Hamiltonian for a Gibbs field on the set of (admissible) coloured graphs gives rise to consistent polygonal fields that enjoy remarkable properties which make them eminently suitable for simulation. Building on the concept of disagreement loops (Schreiber, 2005), Monte Carlo algorithms were developed that allowed for more global updates than the local ones proposed by Clifford \& Nicholls (1994). It was found that the method was robust with respect to noise and able to capture the topology and large- or medium-sized image components well and rapidly; fine details may be lost though, see Kluszczyński et al. $(2005,2007)$.

The purpose of the present paper is to introduce a class of Gibbs-Markov random fields that can be understood as discrete versions of polygonal fields. Our construction is two-staged: first a collection of lines inducing a tessellation of the image is fixed, then a polygonal field is constructed on this tessellation, that is to say, the edges of the field consist of (possibly multiple) segments of the tessellation and the vertices of the field are a subset of the collection of nodes of the tessellation. The generic examples of the tessellationgenerating collection of lines are realisations of the Poisson line process as well as the line sets corresponding to the regular planar lattice, but it is important to stress that these are not the only valid choices. One might for instance pre-process the image to extract the lines across which the gradient changes rapidly. Not every line is equally desired to turn up in the final segmentation. This is taken into account by ascribing a likelihood parameter to each line, reflecting the potential utility of the line in segmentation. For example, lines in dense regions may be down-weighted to give sparse regions a fair chance; alternatively, after pre-processing, the image gradient may guide the choice of weight. As in our previous work, Kluszczyński et al. $(2005,2007)$, the regions of the tessellation are coloured so that the polygonal boundaries of the field coincide with the interfaces separating different colours. Thus, the edges of the field are the maximal linear segments that form the boundaries between different coloured regions, whereas the field vertices are the intersection points between these boundary segments. Note that each edge of the field may contain many tessellation nodes, i.e. intersection points of the tessellation lines.

The analogy with continuum polygonal Markov fields is exploited to define Hamiltonians that are such that desirable properties of these processes (consistency, Markovianity, 
explicit expressions for the partition function) can be carried over to the discrete context. Moreover, the analogy gives rise to new attractive sampling schemes complementing the usual local Gibbs and Metropolis methods employed for Gibbs fields on finite graphs.

The plan of this paper is as follows. In Section 2, we construct a family of admissible coloured polygonal configurations built on regular tessellations, and introduce the concept of a discrete polygonal field. The special class of consistent polygonal fields is treated in detail in Section 3 with emphasis on its dynamic representation. Invariant birth-and-death process dynamics for such consistent polygonal fields are derived in Section 4 exploiting the notion of a disagreement loop. A simple modification for general polygonal fields is the topic of Section 5. More general dynamics are introduced in Section 6 which lead naturally to the path creation and annihilation dynamics that form the topic of Section 7 . In Section 8, the image analysis task of foreground-background separation is recast as a statistical inference problem for a discrete polygonal field model. Examples are presented in Section 9. We conclude with a critical discussion of our approach and indicate some topics that merit further research.

\section{Polygonal fields on regular linear tessellations}

By a regular linear tessellation of the plane we shall understand a countable family $\mathcal{T}$ of straight lines in $\mathbb{R}^{2}$ such that no three lines of $\mathcal{T}$ intersect at one point and such that a bounded subset of $\mathbb{R}^{2}$ is hit by at most a finite number of lines from $\mathcal{T}$, that is to say, $\mathcal{T}$ is locally finite. Even though we will admit $\mathcal{T}$ random in the sequel, it is assumed to be deterministic in this section, an assumption that does not lead to any loss of generality because in case of random $\mathcal{T}$ the construction below can be verbatim repeated realisationwise.

For a bounded open convex set $D \subseteq \mathbb{R}^{2}$ the tessellation $\mathcal{T}$ induces a partition of $D$ into a finite collection $D_{\mathcal{T}}$ of regions of polygonal shapes, possibly chopped off by the boundary. Below, we shall always assume that $\partial D$ contains no nodes of $\mathcal{T}$, defined here as intersection points of lines from $\mathcal{T}$. We shall also require that the intersection of each $l \in \mathcal{T}$ with $\partial D$ consists of exactly two points, i.e. there are no segments of $\partial D$ along lines of $\mathcal{T}$. Consider the set $\hat{\Gamma}_{D}(\mathcal{T})$ of all possible colourings of the regions in $D_{\mathcal{T}}$ into black and white enjoying the additional property that two regions of the same colour can share a node only if either they share a segment or there is a third region of the same colour sharing segments with 
both of them. In other words, there are no regions of the same colour connected only by corners. The family $\Gamma_{D}(\mathcal{T})$ of admissible polygonal configurations in $D$ built on $\mathcal{T}$ is defined to consist of all planar graphs $\gamma$ in $\bar{D}:=D \cup \partial D$ arising as interfaces between black and white regions of colourings $\hat{\gamma} \in \hat{\Gamma}_{D}(\mathcal{T})$. Note that in the sequel we shall consistently use the $\hat{\bullet}$ notation for coloured elements of $\hat{\Gamma}_{D}(\mathcal{T})$ whereas omitting the ${ }^{\wedge}$ will stand for the corresponding colourless contour configuration in $\Gamma_{D}(\mathcal{T})$. Observe that the family $\Gamma_{D}(\mathcal{T})$ could equivalently be defined to consist of all planar graphs $\gamma$ in $D \cup \partial D$ such that

- all edges of $\gamma$ lie on the lines of $\mathcal{T}$,

- all interior vertices of $\gamma$, i.e. those lying in $D$, are of degree 2 ,

- all boundary vertices of $\gamma$, i.e. those lying on $\partial D$, are of degree 1 ,

see also Arak \& Surgailis (1989). In other words, the elements of $\Gamma_{D}(\mathcal{T})$ are collections of disjoint polygonal contours built on $\mathcal{T}$, possibly nested and possibly chopped off by the boundary. Note that by an edge of $\gamma$ we mean a maximal union of connected co-linear tessellation segments in $\gamma$, likewise by a vertex of $\gamma$ we mean a point where two non-co-linear edges of $\gamma$ meet. Thus, the nodes of $\mathcal{T}$ lying in the interior of edges of $\gamma$ are not considered as vertices of $\gamma$, likewise the segments of $\mathcal{T}$ which are not maximal in $\gamma$ are not edges of $\gamma$. To avoid possible ambiguities in the sequel, we shall always use the notions of vertices and edges in the context of the polygonal configurations built on $\mathcal{T}$, whereas the respective terms nodes and segments will be reserved for $\mathcal{T}$. When discussing the relations between polygonal fields and general Gibbs fields below, we will also need a notation for the state space of the latter, which is $\hat{\Omega}_{D}(\mathcal{T})$, standing for the collection of all possible black-white colourings of $D_{\mathcal{T}}$ without the additional requirement of having no corner-only connections. The corresponding family $\Omega_{D}(\mathcal{T})$ of colour-blind contour collections is a superset of $\Gamma_{D}(\mathcal{T})$, as it admits also interior vertices of degree 4 .

Assume now that fixed probability parameters $\pi_{l} \in(0,1)$ are ascribed to the straight lines $l \in \mathcal{T}$. For a function $\mathcal{H}_{D}: \hat{\Gamma}_{D}(\mathcal{T}) \mapsto \mathbb{R} \cup\{+\infty\}$ the (discrete) polygonal field $\hat{\mathcal{A}}_{\mathcal{H}_{D}}$ with Hamiltonian $\mathcal{H}_{D}$ is defined to be the random element in $\hat{\Gamma}_{D}(\mathcal{T})$ such that

$$
\mathbb{P}\left(\hat{\mathcal{A}}_{\mathcal{H}_{D}}=\hat{\gamma}\right)=\frac{\exp \left(-\mathcal{H}_{D}(\hat{\gamma})\right) \prod_{e \in E(\gamma)} \pi_{l[e]}}{\mathcal{Z}\left[\mathcal{H}_{D}\right]},
$$

where $E(\gamma)$ stands for the collection of edges of $\gamma$ considered here to be open, i.e. not to contain their vertices, for formal convenience below, whereas $l[e] \in \mathcal{T}$ is the straight line 
containing $e$, and

$$
\mathcal{Z}\left[\mathcal{H}_{D}\right]:=\sum_{\hat{\theta} \in \hat{\Gamma}_{D}(\mathcal{T})} \exp \left(-\mathcal{H}_{D}(\hat{\theta})\right) \prod_{e \in E(\theta)} \pi_{l[e]}
$$

is the corresponding partition function. Recalling that the Gibbs field $\hat{\mathcal{G}}_{\Psi_{D}}$ on $\hat{\Omega}_{D}(\mathcal{T})$ with Hamiltonian $\Psi_{D}: \hat{\Omega}_{D}(\mathcal{T}) \mapsto \mathbb{R} \cup\{+\infty\}$ is given by

$$
\mathbb{P}\left(\hat{\mathcal{G}}_{\Psi_{D}}=\hat{\gamma}\right)=\frac{\exp \left(-\Psi_{D}(\hat{\gamma})\right)}{\sum_{\hat{\theta} \in \hat{\Omega}_{D}(\mathcal{T})} \exp \left(-\Psi_{D}(\hat{\theta})\right)},
$$

we easily see that the polygonal field $\hat{\mathcal{A}}_{\mathcal{H}_{D}}$ coincides in law with $\hat{\mathcal{G}}_{\Psi_{D}}$ regarded as a $\hat{\Gamma}_{D}(\mathcal{T})$ valued random element for

$$
\Psi_{D}(\hat{\gamma}):= \begin{cases}\mathcal{H}_{D}(\hat{\gamma})-\sum_{e \in E(\gamma)} \log \pi_{l[e]}, & \hat{\gamma} \in \hat{\Gamma}_{D}(\mathcal{T}) \\ +\infty, & \text { otherwise }\end{cases}
$$

In spite of this apparent redundancy there are good reasons for considering the notion of a discrete polygonal field though, one of them being that, unlike (3), the definition (1) admits a natural continuum version and in fact it is the continuum set-up where it has originally arisen, see Arak (1982), Arak \& Surgailis (1989) and Arak et al. (1993). It should also be emphasised that for suitable natural choices of Hamiltonian in (1) the resulting field exhibits striking properties, as will be discussed in Section 3. There is another important reason for introducing (1), however, which is crucial for the purposes of the present paper: there are simulation techniques available for continuum polygonal fields, see Schreiber (2005) and Kluszczyński et al. (2007), whose discrete adaptations can be used to provide new attractive simulation algorithms for discrete Gibbs fields. In the context of image segmentation, the Hamiltonian will include terms that quantify how well a coloured contour collection describes the data (see Section 8 below). To proceed with the presentation of these ideas, we shall begin with a discussion of the so-called consistent polygonal fields and their dynamic representations in Section 3 below.

To complete the present section, we remark that formula (1) can be regarded as the discrete equivalent of the line-based representation for polygonal fields as developed in Arak \& Surgailis (1989). It is natural to ask whether an alternative point-based representation in the spirit of Arak et al. (1993) is available as well. This is indeed the case, but, unlike in the continuum set-up, this alternative representation arises by a simple re-arrangement of the line-indexed product $\prod_{e \in E(\gamma)} \pi_{l[e]}$ as $\left(\prod_{v\left(l_{1}, l_{2}\right) \in V(\gamma) \cap D} \sqrt{\pi_{l_{1}} \pi_{l_{2}}}\right)\left(\prod_{v(l) \in V(\gamma) \cap \partial D} \sqrt{\pi_{l}}\right)$ 
with $v\left(l_{1}, l_{2}\right)$ standing for vertices of $\gamma$ falling into $D$ and arising at the intersection of lines $l_{1}, l_{2} \in \mathcal{T}$, and with $v(l)$ ranging through boundary vertices of $\gamma$ in $\partial D$ lying on $l \in \mathcal{T}$. Thus, in sharp contrast to the continuum case, here the point-based representation does not seem to offer a relevant alternative to the line-based setting.

\section{Consistent polygonal fields on regular tessellations}

In the seminal papers Arak (1982) and Arak \& Surgailis (1989), it was observed that for some particular choices of the Hamiltonian the corresponding polygonal fields enjoy remarkable properties which make them very well suited for simulation - these special processes are the so-called consistent polygonal fields. In the present section, we shall adopt to the discrete case of polygonal fields on regular tessellations the argument of Arak and Surgailis originally developed mainly in the continuum setting (with some exceptions though, see e.g. model D in Surgailis (1991)).

To proceed consider the Hamiltonian

$$
\Phi_{D}(\hat{\gamma}):=-\sum_{e \in E(\gamma)} \sum_{l \in \mathcal{T}, l \nsim e} \log \left(1-\pi_{l}\right)+\sum_{n\left(l_{1}, l_{2}\right) \in \gamma} \log \left(1-\pi_{l_{1}} \pi_{l_{2}}\right), \quad \hat{\gamma} \in \hat{\Gamma}_{D}(\mathcal{T})
$$

with $n\left(l_{1}, l_{2}\right) \in \gamma$ ranging through all nodes of the tessellation $\mathcal{T}$ arising as intersection points of $l_{1}, l_{2} \in \mathcal{T}$ and lying on $\gamma$, that is to say, either lying on the edges of $\gamma$ or coinciding with one of its vertices. Here and in the sequel, $l \nsim e$ means that the line $l$ intersects $e$ but is not co-linear with it. The polygonal field $\hat{\mathcal{A}}_{\Phi_{D}}$ is a consistent polygonal field in the sense made precise by the following theorem.

Theorem 1 The polygonal field $\hat{\mathcal{A}}_{\Phi_{D}}$ with Hamiltonian $\Phi_{D}$ enjoys the following properties:

Consistency: For bounded open convex $D^{\prime} \subseteq D \subseteq \mathbb{R}^{2}$, the field $\hat{\mathcal{A}}_{\Phi_{D}} \cap D^{\prime}$ coincides in law with $\hat{\mathcal{A}}_{\Phi_{D^{\prime}}}$. By increasing $D \uparrow \mathbb{R}^{2}$ this allows us to construct the whole plane extension of the process $\hat{\mathcal{A}}_{\Phi}$ such that $\hat{\mathcal{A}}_{\Phi_{D}}$ coincides in law with $\hat{\mathcal{A}}_{\Phi} \cap D$ for all bounded open convex $D \subseteq \mathbb{R}^{2}$.

Linear sections: For a straight line $l$ containing no nodes of $\mathcal{T}$, the intersection points and intersection directions of $l$ with the edges of the polygonal field $\hat{\mathcal{A}}_{\Phi}$ coincide in distribution with those with the line field $\Lambda_{\mathcal{T}}$ defined to be the random sub-collection of $\mathcal{T}$ where each straight line $l^{*} \in \mathcal{T}$ is chosen to belong to $\Lambda_{\mathcal{T}}$ with probability $\frac{\pi_{l^{*}}}{1+\pi_{l^{*}}}$ and rejected otherwise, and all these choices are made independently. 
Solvability: An explicit formula is available for the partition function:

$$
\mathcal{Z}\left[\Phi_{D}\right]=2\left(\prod_{n\left(l_{1}, l_{2}\right) \in D}\left(1-\pi_{l_{1}} \pi_{l_{2}}\right)\right)^{-1}\left(\prod_{l \in \mathcal{T}, l \cap D \neq \emptyset} \frac{1}{\left(1+\pi_{l}\right)}\right)^{-1}
$$

Markov property: For a smooth closed curve $\theta \subset \mathbb{R}^{2}$ containing no nodes of $\mathcal{T}$, the conditional distribution of $\hat{\mathcal{A}}_{\Phi}$ in the interior of $\theta$ depends on the configuration outside $\theta$ only through the intersection points and intersection directions of $\theta$ with the edges of the polygonal field and through the colouring of the field along $\theta$.

Proof The proof of Theorem 1 is based on the so-called dynamic representation for consistent polygonal fields, being a discrete version of and constructed in full analogy with the corresponding representation in Sections 4 and 5 in Arak \& Surgailis (1989). The idea underlying this construction is to represent the considered polygonal field in terms of the equilibrium evolution of a one-dimensional particle system tracing the polygonal boundaries of the field in two-dimensional time-space. To this end, we interpret the open convex domain $D$ as a set of time-space points $(t, y) \in D$, with $t \in \mathbb{R}$ referred to as the time coordinate and with $y \in \mathbb{R}$ standing for the spatial coordinate of a particle at the time $t$. In this language, a straight line segment in $D$ stands for a piece of the time-space trajectory of a freely moving particle. For a straight line $l$ non-parallel to the spatial axis and crossing the domain $D$ we define in the obvious way its entry point to $D, \operatorname{in}(l, D) \in \partial D$, and its exit point, out $(l, D) \in \partial D$. Without loss of generality we assume that no line of $\mathcal{T}$ is parallel to the spatial axis, possibly rotating the coordinate system if this is not the case.

We choose the time-space birth sites for the new particles by independently placing a birth site

- at each node $n\left(l_{1}, l_{2}\right)$ of the tessellation $\mathcal{T}$ falling into $D$, with probability $\pi_{l_{1}} \pi_{l_{2}}$ (interior birth site),

- at each entry point in $(l, D)$ of lines $l \in \mathcal{T}$ into $D$, with probability $\frac{\pi_{l}}{\left(1+\pi_{l}\right)}$ (boundary birth site).

Each interior birth site $n\left(l_{1}, l_{2}\right)$ emits two particles moving with initial velocities such that the initial segments of their trajectories lie on the lines $l_{1}$ and $l_{2}$ of the tessellation going out from the birth site, unless another particle previously born hits the site in which case the 
birth does not occur. Note that this prevents creation of degree 3 and degree 4 vertices in the resulting graph. Each boundary birth site in $(l, D)$ emits a single particle moving with the initial velocity such that the initial segment of its trajectory lies on $l$ (no precaution similar to the one for interior birth sites above is present because boundary birth sites cannot be hit by previously born particles). All the particles evolve independently in time according to the following rules:

(E1) Between the critical moments listed below, each particle moves with constant velocity so that $d y=v d t$ with $v$ standing for the actual velocity,

(E2) When a particle touches the boundary $\partial D$, it dies,

(E3) In case of a collision of two particles (equal spatial coordinates $y$ at some moment $t$ with $(t, y) \in D)$, both of them die,

(E4) Whenever a particle moving in time-space along $l_{1} \in \mathcal{T}$ reaches a node $n\left(l_{1}, l_{2}\right)$, it changes its velocity so as to move along $l_{2}$ with probability $\pi_{l_{2}}$, and keeps moving along $l_{1}$ otherwise.

The claim constituting the core of the proof is that the union of the time-space trajectories traced by the particles of the above system coincides in distribution with the contour ensemble $\mathcal{A}_{\Phi_{D}}$ of the polygonal field $\hat{\mathcal{A}}_{\Phi_{D}}$, whereas the law of the field itself is recovered by picking one of the two possible black-white colourings at random with probability $1 / 2$. To verify this statement we choose some $\hat{\gamma} \in \hat{\Gamma}_{D}(\mathcal{T})$ and compute the probability that the colour-blind contour ensemble $\gamma$ is traced by the particle system above. To this end, we observe that

- each edge $e \in E(\gamma)$ whose initial (lower time coordinate) vertex lies on $\partial D$ yields a factor $\frac{\pi_{l[e]}}{1+\pi_{l[e]}}$ (boundary birth site) times $\prod_{l \in \mathcal{T}, l \nsim e}\left(1-\pi_{l}\right)$ (no velocity updates along $e)$,

- each of the two edges $e_{1}, e_{2} \in E(\gamma)$ stemming from a common interior birth site $n\left(l_{1}, l_{2}\right)$ yields a factor $\pi_{l_{i}}, i=1,2$, (coming from the birth probability) times $\prod_{i} \prod_{l \in \mathcal{T}, l \nsim e_{i}}\left(1-\pi_{l}\right)$ (no velocity updates along $e_{i}$ ),

- each of the edges $e \in E(\gamma)$ arising due to a velocity update of a particle yields a factor $\pi_{l[e]}$ (velocity update probability) times $\prod_{l \in \mathcal{T}, l \nsim e}\left(1-\pi_{l}\right)$ (no velocity updates along $e$ ), 
- the absence of birth sites in nodes $n\left(l_{1}, l_{2}\right)$ of $\mathcal{T}$ in $D$ not belonging to $\gamma$ yields the factor $\prod_{n\left(l_{1}, l_{2}\right) \in D \backslash \gamma}\left(1-\pi_{l_{1}} \pi_{l_{2}}\right)$ (note that birth sites are allowed in all points of $\gamma-$ either they give rise to particles tracing $\gamma$ or are discarded if hit by a previously born particle),

- the absence of boundary birth sites at those entry points to $D$ of lines of $\mathcal{T}$ which do not give rise to an edge of $\gamma$ yields the factor $\prod_{l \in \mathcal{T}, l \cap D \neq \emptyset, \text { in }(l, D) \notin \gamma} \frac{1}{1+\pi_{l}}$.

Putting these factors together allows us to evaluate the probability of $\gamma$ being traced by the particle system to

$$
\begin{gathered}
\left(\prod_{e \in E(\gamma)} \pi_{l[e]}\right)\left(\prod_{e \in E(\gamma)} \prod_{l \in \mathcal{T}, l \nsim e}\left(1-\pi_{l}\right)\right)\left(\prod_{n\left(l_{1}, l_{2}\right) \in \gamma}\left(1-\pi_{l_{1}} \pi_{l_{2}}\right)\right)^{-1}\left(\prod_{n\left(l_{1}, l_{2}\right) \in D}\left(1-\pi_{l_{1}} \pi_{l_{2}}\right)\right) \times \\
\times\left(\prod_{l \in \mathcal{T}, l \cap D \neq \emptyset} \frac{1}{1+\pi_{l}}\right)=\frac{2 \exp \left(-\Phi_{D}(\hat{\gamma})\right)}{\mathcal{Z}\left[\Phi_{D}\right]} \prod_{e \in E(\gamma)} \pi_{l[e]}
\end{gathered}
$$

with $\mathcal{Z}\left[\Phi_{D}\right]$ given by (6). Taking into account that the choice between the two possible colourings of the field is made with probability $1 / 2$, independently of $\gamma$, we see that the probability of obtaining $\hat{\gamma}$ as the outcome of the particle system evolution is exactly

$$
\frac{\exp \left(-\Phi_{D}(\hat{\gamma})\right)}{\mathcal{Z}\left[\Phi_{D}\right]} \prod_{e \in E(\gamma)} \pi_{l[e]}
$$

and hence the resulting polygonal field coincides in law with $\hat{\mathcal{A}}_{\Phi_{D}}$ as required - this fact will be referred to as the dynamic representation for the polygonal field $\hat{\mathcal{A}}_{\Phi_{D}}$ in the sequel. The Solvability statement (6) follows from the above as well. The remaining properties follow in full analogy with the corresponding argument in Arak \& Surgailis (1989), whence we only provide a brief discussion below. First, the Markov property stated above is a direct consequence of the Gibbsian definition of $\hat{\mathcal{A}}_{\Phi_{D}}$, whereas the Linear sections statement will follow from the form of the boundary birth mechanism described above as soon as we establish the remaining Consistency property. To this end, choose a bounded open convex set $D \subseteq \mathbb{R}^{2}$ and a straight line $l$ intersecting $D$, and define $D^{\prime}$ to be the set of points of $D$ lying to the left of $l$ (lower time coordinates). Clearly then, from the dynamic representation we conclude the Consistency statement for so chosen $D$ and $D^{\prime}$. 
Noting that the dynamic representation is equally available upon rotating the time-space coordinate system, we see that the Consistency holds as well upon cutting off the part of the set $D$ lying to the left of $l$. This means however that the consistency holds upon cutting off pieces of the original set with arbitrary straight lines - a repetitive use of this procedure and a possible passage to the limit allows us to carve from $D$ any arbitrary convex subset. This proves the Consistency claim and completes the proof of Theorem 1.

\section{Disagreement loop birth and death dynamics for consistent polygonal fields}

A crucial concept below will be that of a disagreement loop, borrowed from Schreiber (2005), Section 2.1. This arises from the dynamic construction of the polygonal fields as provided by the evolution rules (E1-4) and the corresponding birth rules specified in the proof of Theorem 1 above.

Suppose that we observe a particular realisation $\gamma \in \Gamma_{D}(\mathcal{T})$ of the colourless contour ensemble $\mathcal{A}_{\Phi_{D}}$ and that we modify the configuration by adding an extra birth site $x_{0}$ to the existing collection of birth sites for $\gamma$ arising in the dynamic representation, while keeping the evolution rules (E1-4) for all the particles, including the two newly added ones if $x_{0}$ is an interior birth site and the single newly added one if $x_{0}$ is a boundary birth site. Denote the resulting new random (colourless) polygonal configuration by $\gamma \oplus x_{0}$. A crucial observation is that, under appropriate coupling of the dynamics of newly added particles with that of the previously existing ones along their sub-trajectories annihilated due to the creation of new particles, for an interior birth site $x_{0}$ the symmetric difference $\gamma \triangle\left[\gamma \oplus x_{0}\right]$ is almost surely a single loop (a closed polygonal curve), possibly self-intersecting and possibly chopped off by the boundary. Likewise, under the same coupling, the symmetric difference $\gamma \triangle\left[\gamma \oplus x_{0}\right]$ is almost surely a single polygon with no self-intersections. We describe this coupling below. It should be noted that for formal convenience we assume that a polygonal configuration $\gamma$ comes together with the knowledge of all attempted birth sites discarded during its creation.

If $x_{0}$ happens to lie on $\gamma$, the new birth attempt gets immediately discarded, yielding $\gamma \triangle\left[\gamma \oplus x_{0}\right]=\emptyset$. Apart from this degenerate case, the leftmost point of the loop $\gamma \triangle\left[\gamma \oplus x_{0}\right]$ is of course $x_{0}$. Each of the two new particles $p_{1}, p_{2}$ emitted from $x_{0}$ move independently, according to $(\mathbf{E} \mathbf{1}-\mathbf{4})$, each giving rise to a disagreement path. The initial segments of 
such a disagreement path correspond to the movement of a particle, say $p_{1}$, before its annihilation in the first collision. If this is a collision with the boundary, the disagreement path gets chopped off and terminates there. If this is a collision with a segment of the original configuration $\gamma$ corresponding to a certain old particle $p_{3}$, the new particle $p_{1}$ dies but the disagreement path continues along the part of the trajectory of $p_{3}$ which is contained in $\gamma$ but not in $\gamma \oplus x_{0}$. A particular case may happen when the collision occurs with a birth site in $\gamma$, where one of the two particles born (denote it by $p_{3}$ ) gets annihilated, whereas the evolution of the second one is re-used under our coupling to yield the extension of the evolution of $p_{1}$ - we do so instead of annihilating both particles emitted from the birth site and building the extension of the evolution of $p_{1}$ anew. The choice of the annihilated and surviving particles upon such a collision at a node $n\left(l_{1}, l_{2}\right)$ with the current direction of $p_{1}$ being $l_{1}$ is made as follows: with probability $\left(1-\pi_{l_{2}}\right)$ we annihilate the particle moving along $l_{2}$ (no velocity update for $p_{1}$ ) and with probability $\pi_{l_{2}}$ we annihilate the particle moving along $l_{1}$ ( $p_{1}$ undergoes a velocity update). Clearly, this is consistent with the dynamic representation. Thus, we note that under the so-constructed coupling $p_{1}$ always moves according to the usual evolution rules (E1-4) because the particles emitted by the hit birth site did so. At some further moment $p_{3}$ dies itself in $\gamma$, touching the boundary or killing another particle $p_{4}$ in $\gamma$. In the second case, however, this collision only happens for $\gamma$ and not for $\gamma \oplus x_{0}$, so the particle $p_{4}$ survives (for some time) in $\gamma \oplus x_{0}$ yielding a further connected portion of the disagreement path for $p_{1}$, which is contained in $\gamma \oplus x_{0}$ but not in $\gamma$. Likewise, it may happen that $p_{3}$ reaches a node $n\left(l_{1}, l_{2}\right)$ where a birth attempt was made in $\gamma$ but was discarded due to the presence of $p_{3}$. Now that $p_{3}$ is absent in $\gamma \oplus x_{0}$, this birth does occur for $\gamma \oplus x_{0}$. We require in our coupling that one of the emitted particles follow the remaining trajectory of $p_{3}$ in $\gamma$, thus re-using for the new particle the random choices made before for $p_{3}$. Clearly, the second particle emitted by the birth site denoted by $p_{4}$ as above, adds one further sub-portion of the disagreement loop in $\left[\gamma \oplus x_{0}\right] \backslash \gamma$. Again, the particle evolving according to the previous trajectory of $p_{3}$ moves in accordance with all the rules of the dynamic representation because so did $p_{3}$.

A recursive continuation of this construction shows that the disagreement path initiated by $p_{1}$ consists alternately of connected polygonal sub-paths contained in $\left[\gamma \oplus x_{0}\right] \backslash \gamma$ (call these positive parts) and in $\gamma \backslash\left[\gamma \oplus x_{0}\right]$ (call these negative parts). Note that this disagreement path is self-avoiding and, in fact, it can be represented as the graph of some piecewise linear function $t \mapsto y(t)$. Clearly, the same applies for the disagreement path initiated by 
the second initial particle $p_{2}$. An important observation is that whenever two positive or two negative parts of the two disagreement paths hit each other, both disagreement paths may die at this point and the disagreement loop may close (as opposed to intersections of segments of distinct signs which do not have this effect). Obviously, if the disagreement loop does not close in the above way, it gets eventually chopped off by the boundary. Note that upon the intersection of two positive or negative sub-paths at a node $v$, instead of getting killed a disagreement loop may also continue due to altering the status of a birth site, should it occur at $v$. Indeed, for negative sub-paths a birth site previously discarded may be reactivated, whereas for positive sub-paths a birth site may be inactivated.

We shall write $\Delta^{\oplus}\left[x_{0} ; \gamma\right]=\gamma \Delta\left[\gamma \oplus x_{0}\right]$ to denote the (random) disagreement loop constructed above. It remains to consider the case of $x_{0}$ being a boundary birth site, which is much simpler because there is only one particle emitted and so, under our coupling, $\Delta^{\oplus}\left[x_{0} ; \gamma\right]=\gamma \triangle\left[\gamma \oplus x_{0}\right]$ is easily seen to be a single self-avoiding polygonal path eventually chopped off by the boundary. We abuse the language and call such $\Delta^{\oplus}\left[x_{0} ; \gamma\right]$ a (degenerate) disagreement loop as well.

Likewise, a disagreement loop arises if we remove one birth site $x_{0}$ from the collection of birth sites of an admissible polygonal configuration $\gamma \in \Gamma_{D}(\mathcal{T})$, while keeping the evolution rules for all the remaining particles. We write $\gamma \ominus x_{0}$ for the configuration obtained from $\gamma$ by removing $x_{0}$ from the list of the birth sites, while the resulting random disagreement loop is denoted by $\Delta^{\ominus}\left[x_{0} ; \gamma\right]$, so that $\Delta^{\ominus}\left[x_{0} ; \gamma\right]=\gamma \Delta\left[\gamma \ominus x_{0}\right]$. Note that again an empty disagreement loop may occur, should we annihilate a birth site where the birth attempt was discarded due to the presence of previously created particles. We refer the reader to Schreiber (2005) for further formal details of the disagreement loop concept.

With the above terminology we are in a position to describe random dynamics on the coloured configuration space $\hat{\Gamma}_{D}(\mathcal{T})$ which leave invariant the law of the polygonal field $\hat{\mathcal{A}}_{\Phi_{D}}$. Particular care is needed, however, to distinguish between the notion of time considered in the dynamic representation of the polygonal field as well as throughout the construction of the disagreement loops above, and the notion of time to be introduced for the random dynamics on $\hat{\Gamma}_{D}(\mathcal{T})$ constructed below. To make this distinction clear we shall refer to the former as to the representation time (r-time for short) and shall keep for it the notation $t$, while the latter will be called the simulation time (s-time for short) and will be denoted by $s$ in the sequel.

Consider the following pure jump birth-and-death type Markovian (DL)-dynamics on 
$\hat{\Gamma}_{D}(\mathcal{T})$

DL:birth At each $x:=n\left(l_{1}, l_{2}\right) \in D, l_{1}, l_{2} \in \mathcal{T}$, with intensity $\pi_{l_{1}} \pi_{l_{2}} d s$ set $\gamma_{s+d s}:=\gamma_{s} \oplus x$ (interior births), then construct $\hat{\gamma}_{s+d s}$ by randomly choosing, with probability $1 / 2$, either of the two possible colourings for $\gamma_{s+d s}$. Proceed likewise at each $x:=l \cap \partial D, l \in$ $\mathcal{T}$, with intensity $\frac{\pi_{l}}{1+\pi_{l}} d s$ (boundary births).

DL:death For each interior and boundary birth site $x$ in $\gamma_{s}$, with intensity 1 set $\gamma_{s+d s}:=$ $\gamma_{s} \ominus x$, then construct $\hat{\gamma}_{s+d s}$ by randomly choosing, with probability $1 / 2$, either of the two possible colourings for $\gamma_{s+d s}$.

If none of the above updates occurs, we keep $\hat{\gamma}_{s+d s}=\hat{\gamma}_{s}$. It is convenient to perceive the above dynamics in terms of generating random disagreement loops $\lambda$ and setting $\gamma_{s+d s}:=$ $\gamma_{s} \Delta \lambda$, with the loops of the type $\Delta^{\oplus}[\cdot ; \cdot]$ corresponding to the rule DL:birth and $\Delta^{\ominus}[\cdot ; \cdot]$ to the rule DL:death.

As a consequence of the dynamic representation developed in the proof of Theorem 1 we obtain

Proposition 1 The distribution of the consistent polygonal field $\hat{\mathcal{A}}_{\Phi_{D}}$ is the unique invariant law of the dynamics given by DL:birth and DL:death. The resulting stationary process is reversible. Moreover, for any initial distribution of $\hat{\gamma}_{0}$ the laws of the random polygonal fields $\hat{\gamma}_{s}$ converge in variational distance to the law of $\hat{\mathcal{A}}_{\Phi_{D}}$ as $s \rightarrow \infty$.

Proof To establish the invariance, we note first that the DL dynamics is easily seen to preserve the Bernoulli law imposed by the dynamic representation on the collection of birth sites of the process (including the birth attempt sites discarded due to the incidence with previously born particles). Moreover, the DL dynamics is explicitly constructed so as to ensure that if the original configuration is traced by particles evolving according to the rules of the dynamic construction and emitted from the given collection of birth sites, then so is the updated configuration, with the accordingly updated collection of birth sites. This ensures the required invariance.

The reversibility of the dynamics follows from the obvious reversibility of the birth site birth-and-death process. To see this, assign to each birth site (including the discarded birth attempts) the (random) sequence of velocity update choices to be made in the course of evolution of the particles it emits (would emit), which corresponds to the full knowledge of 
the (unlimited) particle evolution. This makes birth sites into birth packages, algorithmically representable e.g. by assigning to each birth site the corresponding seed for random number generation. Thus, we end up with a random collection of birth packages containing all the randomness of the field, that is to say, fully determining the resulting field now obtainable by a deterministic procedure. For definiteness, we assume that if a particle passes on its way a birth site, which thus becomes inactivated, starting from the next evolution step after this event the particle evolves further according to the birth package of the just inactivated birth site rather than according to its own. Now, in this context it is easy to check that the reverse move to adding a birth package at $x_{0}$ is removing the same birth package at $x_{0}$ and vice versa, that is to say $\left[\gamma \oplus x_{0}\right] \ominus x_{0} \equiv \gamma$ and $\left[\gamma \ominus x_{0}\right] \oplus x_{0} \equiv \gamma$ with $x_{0}$ carrying always the same birth package. This ensures the reversibility under the above birth package interpretation. To get the required reversibility for polygonal configurations not containing the full knowledge of entire birth packages (unnecessarily determining the unlimited extension of the trajectories of annihilated particles as if they were to survive forever) is easily recovered by integrating out the spurious components of birth packages.

The uniqueness and convergence statements in the above proposition require a short justification as well. They both follow from the observation that, in finite volume, regardless of the initial state, the process $\hat{\gamma}_{s}$ spends a non-null fraction of time in the state 'black' (no contours, the whole domain $D$ coloured black). Indeed, this observation allows us to conclude the required uniqueness and convergence by a standard coupling argument, e.g. along the lines of the proof of Theorem 1.2 in Liggett (1985).

\section{Disagreement loop birth and death dynamics for general polygonal fields}

Take now a general polygonal field $\hat{\mathcal{A}}_{\mathcal{H}_{D}+\Phi_{D}}$ with a Hamiltonian $\mathcal{H}_{D}+\Phi_{D}: \hat{\Gamma}_{D}(\mathcal{T}) \rightarrow$ $\mathbb{R} \cup\{+\infty\}$ as in (1). Consider the following modification of the basic (DL) dynamics constructed in Section 4 above:

$\mathrm{DL}[\mathbf{H}]:$ birth At each $x:=n\left(l_{1}, l_{2}\right) \in D, l_{1}, l_{2} \in \mathcal{T}$, with intensity $\pi_{l_{1}} \pi_{l_{2}} d s$ set $\delta_{s+d s}:=$ $\gamma_{s} \oplus x$, whereupon construct $\hat{\delta}$ by choosing with probability $1 / 2$ one of the two possible colourings for $\delta$. Then, with probability $\min \left(1, \exp \left[\mathcal{H}_{D}\left(\hat{\gamma}_{s}\right)-\mathcal{H}_{D}(\hat{\delta})\right]\right)$, put $\hat{\gamma}_{s+d s}:=\hat{\delta}$, otherwise keep $\hat{\gamma}_{s+d s}:=\hat{\gamma}_{s}$. Proceed likewise at each $x:=l \cap \partial D, l \in \mathcal{T}$, with intensity $\frac{\pi_{l}}{1+\pi_{l}} d s$. 
$\mathbf{D L}[\mathbf{H}]$ :death for each interior and boundary birth site $x$ in $\gamma_{s}$, with intensity $1 \cdot d s$ set $\delta:=\gamma_{s} \ominus x$, whereupon construct $\hat{\delta}$ by choosing with probability $1 / 2$ one of the two possible colourings for $\delta$. Then, with probability $\min \left(1, \exp \left[\mathcal{H}_{D}\left(\hat{\gamma}_{s}\right)-\mathcal{H}_{D}(\hat{\delta})\right]\right)$, put $\hat{\gamma}_{s+d s}:=\hat{\delta}$, otherwise keep $\hat{\gamma}_{s+d s}:=\hat{\gamma}_{s}$.

In other words, the original dynamics DL are used to propose a new configuration $\hat{\delta}$, which is then accepted with probability $\min \left(1, \exp \left[\mathcal{H}_{D}\left(\hat{\gamma}_{s}\right)-\mathcal{H}_{D}(\hat{\delta})\right]\right)$, and rejected otherwise. By a straightforward verification of the detailed balance conditions and an appeal to Proposition 1 , we obtain the following result.

Theorem 2 The distribution of the polygonal field $\hat{\mathcal{A}}_{\mathcal{H}_{D}+\Phi_{D}}$ given by (1) is the unique invariant law of the dynamics described by $\mathbf{D L}[\mathbf{H}]: \mathbf{b i r t h}$ and $\mathbf{D L}[\mathbf{H}]: \mathbf{d e a t h}$. The resulting stationary process is reversible. Moreover, for any initial distribution of $\hat{\gamma}_{0}$, the laws of the random polygonal fields $\hat{\gamma}_{s}$ converge in total variation to the law of $\hat{\mathcal{A}}_{\mathcal{H}_{D}+\Phi_{D}}$ as $s \rightarrow \infty$.

\section{Generalised dynamic representation for consistent polygonal fields}

The dynamic construction of consistent polygonal fields borrowed from Arak \& Surgailis (1989) and adapted for tessellation-based fields in the proof of Theorem 1 can be regarded as revealing increasing portions of the polygonal field in the course of time flow. Under this interpretation, with probability 1 the portion of a polygonal field in a bounded open convex domain $D$ uncovered by time $t$ is, upon closure, precisely the closure of its intersection with $D_{t}=\bar{D} \cap(-\infty, t] \times \mathbb{R}_{+}$. The idea underlying our generalised dynamic representation developed in the present section below is to replace the above family $D_{t}$ by some other timeincreasing family of subsets of $D$, also denoted by $D_{t}$ in the sequel, eventually covering the whole $D$, and to try to provide a natural construction of the polygonal field being gradually uncovered on the growing domain $D_{t}$ in the course of time flow. We shall always assume that $D_{t}$ is convex, for otherwise we would have to deal with situations where two or more disconnected parts of an edge of the field have been revealed, which leads to unwanted dependencies along the segments connecting these parts. Taking this into account, and having formal convenience in mind, we impose the following natural assumptions on $D_{t}, t \in[0,1]$,

- $\left(D_{t}\right)_{t \in[0,1]}$ is an increasing family of compact convex subsets of $\bar{D}=D \cup \partial D$, 
- $D_{0}$ is a single point $x$ in $\bar{D}=D \cup \partial D$,

- $D_{1}$ coincides with $\bar{D}$,

- $D_{t}$ is continuous in the usual Hausdorff metric,

- For each $l \in \mathcal{T}$ the intersection $l \cap D_{\tau_{l}}$ consists of exactly one point $\mathbb{A}(l)$, where $\tau_{l}:=\inf \left\{t \in[0,1], D_{t} \cap l \neq \emptyset\right\}$. Moreover, $\mathbb{A}(l)$ is not a node of $\mathcal{T}$.

The condition requiring that $D_{0}$ be a singleton can be easily weakened, in fact it is enough if $D_{0}$ be a linear segment, yet this requires certain technical changes in the dynamics below without providing essential generalisations and hence we do not discuss this option here. The point $\mathbb{A}(l)$ will be referred to as the anchor point for $l$, this induces the anchor mapping $\mathbb{A}: \mathcal{T} \rightarrow \bar{D}$. Consider now the following dynamics in time $t \in[0,1]$, with all updates given by the rules below performed independently of each other.

(GE:Initialise) Begin with an empty field at time 0,

(GE1) Between critical moments listed below, during the time interval $[t, t+d t]$, the field lines in $D_{t}$ hitting $\partial D_{t}$ extend straight to $D_{t+d t} \backslash D_{t}$,

(GE2) When a field line hits the boundary $\partial D$, its stops growing in this direction (recall that $\partial D$ contains no segments along lines of $\mathcal{T}$ and so the intersection of a field line with $\partial D$ consists of at most two points),

(GE3) When two field lines intersect in $D_{t+d t} \backslash D_{t}$, they are not extended any further beyond the intersection point (stop growing in the direction marked by the intersection point),

(GE4) Whenever a node $n\left(l_{1}, l_{2}\right), l_{1}, l_{2} \in \mathcal{T}$, falls into $D_{t+d t} \backslash D_{t}$, and $l_{1}$ is a current field line hitting $\partial D_{t}$, with probability $\pi_{l_{2}}$ we update the direction of the line to $l_{2}$, extending away from the anchor point $\mathbb{A}\left(l_{2}\right)$. We keep the previous direction along $l_{1}$ otherwise. Should several such vertices belong to $D_{t+d t} \backslash D_{t}$, the direction updates are performed independently,

(GE:LineBirth) Whenever an anchor point $\mathbb{A}(l), l \in \mathcal{T}$, falls into $D_{t+d t} \backslash D_{t}$, with probability $\frac{\pi_{l}}{1+\pi_{l}}$ a new field line $l$ is born at $\mathbb{A}(l)$, extending in both directions, 
(GE:VertexBirth) Whenever a node $n\left(l_{1}, l_{2}\right), l_{1}, l_{2} \in \mathcal{T}$, falls into $D_{t+d t} \backslash D_{t}$, with probability $\pi_{l_{1}} \pi_{l_{2}}$ two new field lines $l_{1}$ and $l_{2}$ are born, each extending in the direction away from its anchor point, unless another field line present at time $t$ hits $n\left(l_{1}, l_{2}\right)$ in which case the birth does not occur.

It is worth noting that if we choose the family $D_{t}$ so that $D_{t}:=\bar{D} \cap\left(-\infty,(1-t) x_{\min }+\right.$ $\left.t x_{\max }\right] \times \mathbb{R}_{+}$, where $x_{\min }$ and $x_{\max }$ are the minimal and maximal x-coordinates of points in $D$ (assume that $\bar{D}$ contains exactly one point with x-coordinate $x_{\min }$ for formal convenience), the generalised dynamic representation (GE) coincides with the original Arak \& Surgailis one determined by rules (E1-4) and we have $\mathbb{A}(l)=\operatorname{in}(l, D)$.

In analogy with the corresponding result for the usual dynamic construction, as established in the proof of Theorem 1, we show that the field resulting from the above (GE) construction does coincide in law with the contour ensemble $\mathcal{A}_{\Phi_{D}}$ and, consequently, with $\hat{\mathcal{A}}_{\Phi_{D}}$ upon choosing with probability $1 / 2$ one of the two possible colourings.

Theorem 3 The random contour ensemble resulting from the above construction (GE) coincides in law with $\mathcal{A}_{\Phi_{D}}$.

Proof To verify the statement of the theorem, we choose some $\gamma \in \Gamma_{D}(\mathcal{T})$ and compute the probability that the colour-blind contour ensemble $\gamma$ is obtained as a result of the above construction. To this end, we observe that

- each edge $e \in E(\gamma)$ containing the anchor point $\mathbb{A}(l[e])$ in its interior yields a factor $\frac{\pi_{l[e]}}{1+\pi_{l[e]}}\left(\right.$ line birth) times $\prod_{l \in \mathcal{T}, l \nsim e}\left(1-\pi_{l}\right)$ (no direction updates along $e$ ),

- each of the two edges $e_{1}, e_{2} \in E(\gamma)$ stemming from a common interior birth site $n\left(l_{1}, l_{2}\right)$ yields a factor $\pi_{l_{i}}, i=1,2$, (coming from the birth probability) times $\prod_{i} \prod_{l \in \mathcal{T}, l \nsim e}\left(1-\pi_{l}\right)$ (no direction updates along $e_{i}$ ),

- each of the edges $e \in E(\gamma)$ arising due to a direction update yields a factor $\pi_{l[e]}$ (direction update probability) times $\prod_{l \in \mathcal{T}, l \text { }_{e}}\left(1-\pi_{l}\right)$ (no direction updates along $e$ ),

- the absence of birth sites at nodes $n\left(l_{1}, l_{2}\right)$ of $\mathcal{T}$ in $D$ not belonging to $\gamma$ yields the factor $\prod_{n\left(l_{1}, l_{2}\right) \in D \backslash \gamma}\left(1-\pi_{l_{1}} \pi_{l_{2}}\right)$ (note that birth sites are allowed in all points of $\gamma-$ either they give rise to lines tracing $\gamma$ or are discarded if hit by a previously born line), 
- the absence of line births at those anchor points $\mathbb{A}(l), l \in \mathcal{T}$, which do not give rise to an edge of $\gamma$ yields the factor $\prod_{l \in \mathcal{T}, l \cap D \neq \emptyset, \mathbb{A}(l) \notin \gamma} \frac{1}{1+\pi_{l}}$.

Putting these factors together allows us to evaluate the probability of $\gamma$ arising as a result of the (GE) construction as

$$
\begin{gathered}
\left(\prod_{e \in E(\gamma)} \pi_{l[e]}\right)\left(\prod_{e \in E(\gamma)} \prod_{l \in \mathcal{T}, l \nsim e}\left(1-\pi_{l}\right)\right)\left(\prod_{n\left(l_{1}, l_{2}\right) \in \gamma}\left(1-\pi_{l_{1}} \pi_{l_{2}}\right)\right)^{-1}\left(\prod_{n\left(l_{1}, l_{2}\right) \in D}\left(1-\pi_{l_{1}} \pi_{l_{2}}\right)\right) \times \\
\times\left(\prod_{l \in \mathcal{T}, l \cap D \neq \emptyset} \frac{1}{1+\pi_{l}}\right)=\frac{2 \exp \left(-\Phi_{D}(\hat{\gamma})\right)}{\mathcal{Z}\left[\Phi_{D}\right]} \prod_{e \in E(\gamma)} \pi_{l[e]}
\end{gathered}
$$

in full analogy to $(7)$ with $\mathcal{Z}\left[\Phi_{D}\right]$ given by (6). This completes the proof as in the argument establishing the dynamic representation in Theorem 1.

\section{$7 \quad$ Path creation and annihilation dynamics for general polygonal fields}

The purpose of the present section is to use the generalised dynamic representation to construct dynamics on the space of polygonal configurations in a spirit similar to the derivation of the disagreement loop dynamics from the basic Arak \& Surgailis dynamic representation discussed in Section 4 above. This means in particular that the path creation and annihilation procedure is a direct generalisation of the disagreement loop dynamics; there are good reasons, though, to treat the disagreement loop procedure separately, since it is usually less complex from a computational viewpoint due to the particularly simple nature of the anchor mapping $\mathbb{A}(l)=$ in $(l, D)$. Also, as we shall see below, the main advantage of choosing the family $D_{t}$ different from the one corresponding to the Arak \& Surgailis construction relies on the particular form of line birth events, whereas the vertex birth Monte-Carlo moves are equally well generated by the simpler disagreement loop dynamics. It should be noted here that further interest in the generalised dynamic representation stems from its theoretical implications (Schreiber, work in progress), giving an insight into the higher order correlation structure of the field, which falls beyond the scope of the present application-oriented paper. 
To proceed towards the so posed objective, we note first that, in full analogy with the DL dynamics above, adding a new line birth site for $l \in \mathcal{T}$ at its anchor point $l$ as specified in (GE:LineBirth) and letting it evolve thereupon according to the rules of the (GE) dynamics under the same coupling as for DL above, yields a disagreement path as the symmetric difference between the previous onfiguration $\gamma$ and the new one $\gamma \oplus_{D_{t}} l$, where $\oplus_{D_{t}}$ is the line creation operator corresponding to the choice of $\left(D_{t}\right)_{t \in[0,1]}$. Likewise, annihilating a line birth site, with the corresponding operator denoted by $\ominus_{D_{t}}$, yields under the usual coupling a disagreement path as well. Clearly, disagreement paths or loops are also obtained upon adding or removing a vertex, with the corresponding operators denoted again by $\oplus_{D_{t}}$ and $\ominus_{D_{t}}$ with no ambiguity arising between the line creation and annihilation operators which can be distinguished by the different nature of their right argument. Consider the following path creation and annihilation (PCA) dynamics:

PCA:CreateLine For each $l \in \mathcal{T}$, with intensity $\frac{\pi_{l}}{1+\pi_{l}} d s$, set $\gamma_{s+d s}:=\gamma_{s} \oplus_{D_{t}} l$,

PCA:AnnihilateLine For each $l \in \mathcal{T}$ such that $\mathbb{A}(l) \in \gamma$, with intensity 1 , set $\gamma_{s+d s}:=$ $\gamma_{s} \ominus_{D_{t}} l$

PCA:CreateVertex For each $x:=n\left(l_{1}, l_{2}\right) \in D, l_{1}, l_{2} \in \mathcal{T}$, with intensity $\pi_{l_{1}} \pi_{l_{2}} d s$, set $\gamma_{s+d s}:=\gamma_{s} \oplus_{D_{t}} x$

PCA:AnnihilateVertex For each interior birth site $x$ in $\gamma_{s}$, with intensity 1 , set $\gamma_{s+d s}:=$ $\gamma_{s} \ominus_{D_{t}} x$

In full analogy with Proposition 1 we get

Proposition 2 The distribution of the consistent colour-blind polygonal field $\mathcal{A}_{\Phi_{D}}$ is the unique invariant law of the dynamics PCA. The resulting stationary process is reversible. Moreover, for any initial distribution of $\gamma_{0}$ the laws of the random polygonal fields $\gamma_{s}$ converge in variational distance to the law of $\mathcal{A}_{\Phi_{D}}$ as $s \rightarrow \infty$.

Clearly, a version of the above dynamics can be constructed for general polygonal fields with Hamiltonian $\Phi_{D}+\mathcal{H}_{D}$ for some $\mathcal{H}_{D}: \hat{\Gamma}_{D}(\mathcal{T}) \rightarrow \mathbb{R}_{+}$. To this end, we regard the PCA dynamics as a generator of update proposals, which are then accepted with probability 1 if $\mathcal{H}_{D}$ decreases and with probability $\exp \left(-\Delta \mathcal{H}_{D}\right)$ otherwise, where $\left.\Delta \mathcal{H}_{D}\right)$ is the increase in $\mathcal{H}_{D}$. To put it in formal terms, proceed as follows: 
PCA $[\mathbf{H}]$ Given $\hat{\gamma}_{s}$ generate an update proposal $\delta$ for $\gamma_{s+d s}$, colour it randomly in one of two possible ways with probability $1 / 2$ each, thus obtaining the coloured update proposal $\hat{\delta}$. Now, if $\mathcal{H}_{D}(\hat{\delta})<\mathcal{H}_{D}\left(\hat{\gamma}_{s}\right)$, set $\hat{\gamma}_{s+d s}:=\hat{\delta}$, otherwise set $\hat{\gamma}_{s+d s}:=\hat{\delta}$ with probability $\exp \left(-\left[\mathcal{H}_{D}(\hat{\delta})-\mathcal{H}_{D}\left(\hat{\gamma}_{s}\right)\right]\right)$ and keep $\hat{\gamma}_{s+d s}=\hat{\gamma}_{s}$ with the complementary probability.

Using Proposition 2 and verifying the usual detailed balance conditions we come to

Theorem 4 The distribution of the polygonal field $\hat{\mathcal{A}}_{\mathcal{H}_{D}+\Phi_{D}}$ is the unique invariant law of the dynamics $\mathbf{P C A}[\mathbf{H}]$. The resulting stationary process is reversible. Moreover, for any initial distribution of $\hat{\gamma}_{0}$ the laws of the random polygonal fields $\hat{\gamma}_{s}$ converge in variational distance to the law of $\hat{\mathcal{A}}_{\Phi_{D}+\mathcal{H}_{D}}$ as $s \rightarrow \infty$.

\section{Image segmentation as a statistical inference prob- lem}

Here we recall the framework for image interpretation in terms of planar Markov random fields (see e.g. Chellapa \& Jain (1993)).

The data consist of some discretised grey level image. Write $S$ for the set of sites and $L=\{0, \cdots, 255\}$ for the label set. Formally, the data is a vector $y=\left(y_{s}\right)_{s \in S}$ with $y_{s} \in L$. As before, assume that $D$ is a bounded, convex and open subset of $\mathbb{R}^{2}$. We will impose the further constraint that $D$ contains $S$.

Below, we shall reformulate the task of segmenting $y$ into foreground and background regions as a statistical parameter estimation problem. Since the aim is to interpret $y$ in terms of a black-and-white coloured polygonal configuration, we seek to find a $\hat{\gamma}^{*} \in \hat{\Gamma}_{D}$ that explains the data 'best' in the sense of having a small misclassification rate as well as exhibiting desirable 'smoothness' properties. More specifically, we shall base inference on the polygonal field distribution

$$
\frac{\exp \left(-Y_{D, S}^{y, \beta}(\hat{\gamma})\right)}{\mathcal{Z}\left[Y_{D, S}^{y, \beta}\right]} \prod_{e \in E(\gamma)} \pi_{l[e]}
$$

with

$$
Y_{D, S}^{y, \beta}(\hat{\gamma})=\Phi_{D}(\hat{\gamma})+\beta X_{D, S}(\hat{\gamma} ; y), \quad \hat{\gamma} \in \hat{\Gamma}_{D}(\mathcal{T})
$$


for $\beta>0$, and partition function

$$
\mathcal{Z}\left[Y_{D, S}^{y, \beta}\right]=\sum_{\hat{\theta} \in \Gamma_{D}(\mathcal{T})} \exp \left(-Y_{D, S}^{y, \beta}(\hat{\theta})\right) \prod_{e \in E(\theta)} \pi_{l[e]} .
$$

Thus, (9) is a of the form (1) with a Hamiltonian that is a weighted sum of two terms: $\Phi_{D}(\hat{\gamma})$ given by $(5)$, and $X_{D, S}(\hat{\gamma} ; y)$ that describes the goodness-of-fit between the coloured configuration $\hat{\gamma}$ and the data $y$. In the examples to be presented in Section 9, we shall use the 'misclassification rate'

$$
X_{D, S}(\hat{\gamma} ; y)=\sum_{s \in S}\left|y_{s}-\hat{\gamma}_{s}\right|
$$

where $\hat{\gamma}_{s}$ denotes the colour of $\hat{\gamma}$ at pixel $s$. Note that as $D$ contains $S, \hat{\gamma}_{s}$ is well-defined. Moreover, observe that (9) can also be regarded as the distribution of a classical Gibbs field on $\hat{\Omega}_{D}(\mathcal{T})$, the space consisting of all binary colourings of $D_{\mathcal{T}}$ with Hamiltonian of the form (4),

$$
\Psi_{D}(\hat{\gamma})= \begin{cases}Y_{D, S}^{y, \beta}(\hat{\gamma})-\sum_{e \in E(\gamma)} \log \pi_{l[e]}, & \hat{\gamma} \in \hat{\Gamma}_{D}(\mathcal{T}) \\ +\infty, & \text { otherwise }\end{cases}
$$

In conclusion, we place ourselves in a regularisation framework in which a likelihood term $X_{D, S}(\hat{\gamma} ; y)$ is weighted against a regularisation term $\Phi_{D}(\hat{\gamma})$ on coloured polygonal configurations and line desirability terms $-\sum_{e \in E(\gamma)} \log \pi_{l[e]}$. Upon observation of $y$, the goal is to estimate the underlying coloured polygonal configuration by Hamiltonian optimisation. More specifically, we use the simulated annealing algorithm (Geman \& Geman (1984), Haario and Saksman (1991)) and let $\beta \uparrow \infty$ to find the polygonal configurations $\hat{\gamma}^{*}$ having minimal misclassification rate while keeping the other terms in the Hamiltonian (10) fixed.

\section{$9 \quad$ Examples}

In this section we present results produced by our $\mathrm{C}++$ implementation of the above algorithms on toy and real life examples. The model (9) was used with components (5) and (11). We used a combination of the $\mathbf{D L}[\mathbf{H}]$ :birth-death algorithm of Section 5 and the PCA $[\mathbf{H}]$ dynamics of Section 7 combined with the usual local basic polygon recolourings constituting the core of the standard simulation algorithms for lattice-indexed Gibbs-Markov fields (see e.g. Winkler (2003)). Path creation and annihilation, being global in nature, is useful in the beginning of the simulated annealing procedure, as are 
the disagreement loop updates. In later stages of the simulation they are complemented with local polygon updates to take care of fine details.

We applied simulated annealing, treating (5) as the Hamiltonian of the reference distribution, and a linear cooling schedule that amounts to setting $\beta$ equal to the number of iterates divided by 20 . At the present stage, for the family $\mathcal{T}$ we chose the regular square lattice, yet it should be emphasised that experiments with other lattices are underway.

We first tested the method on synthetic images. At each iteration, the disagreement loop dynamics were chosen with probability 0.7 , path creation and annihilation with probability 0.1 , leaving probability 0.2 for local polygon updates. For the path creation and annihilation dynamics we set the family $D_{t}$ to grow from a point along a randomly directed segment until reaching the boundary $\partial D$ and thereupon to extend in perpendincular direction until covering the whole of $D$. Also, random rotations of the time axis for the disagreement loop dynamics were performed every 1000 moves.

Figure 1 (left) shows a blurry 'A' overlaid on the segmentation result obtained after 50, 000 steps; Figure 1 (right) is the result after 75, 000 iterations on a 'B'. In both cases, about a minute computation time was needed on a state of the art desktop computer to separate foreground from background. The target misclassification rate is 0.04 for both figures.
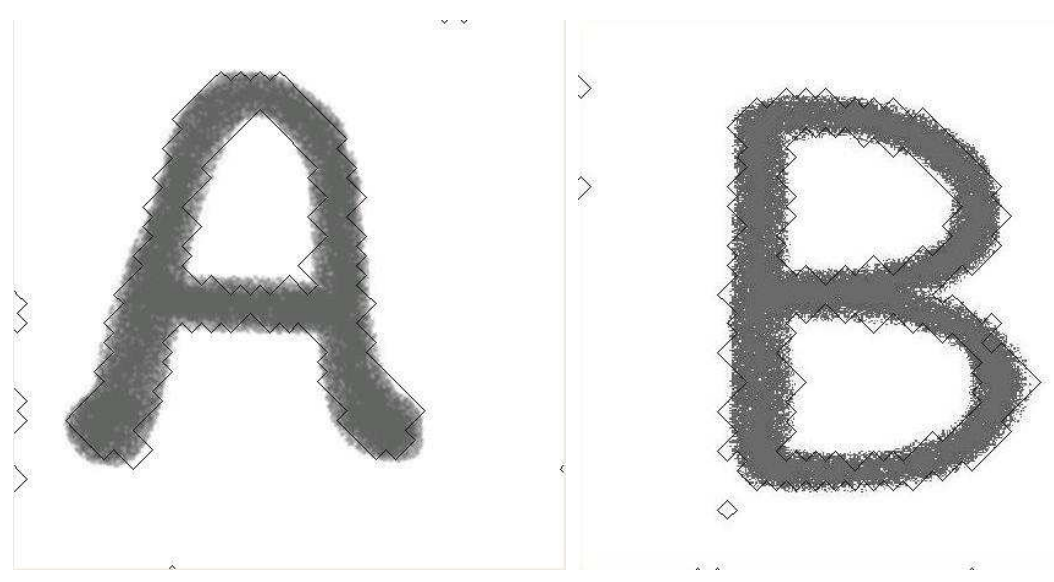

Figure 1: Segmented letters: A (left) and B (right).

Also we used grey level images from the Berkeley Segmentation Dataset and Benchmark web site

http://www. eecs. berkeley.edu/Research/Projects/CS/vision/grouping/segbench/ 

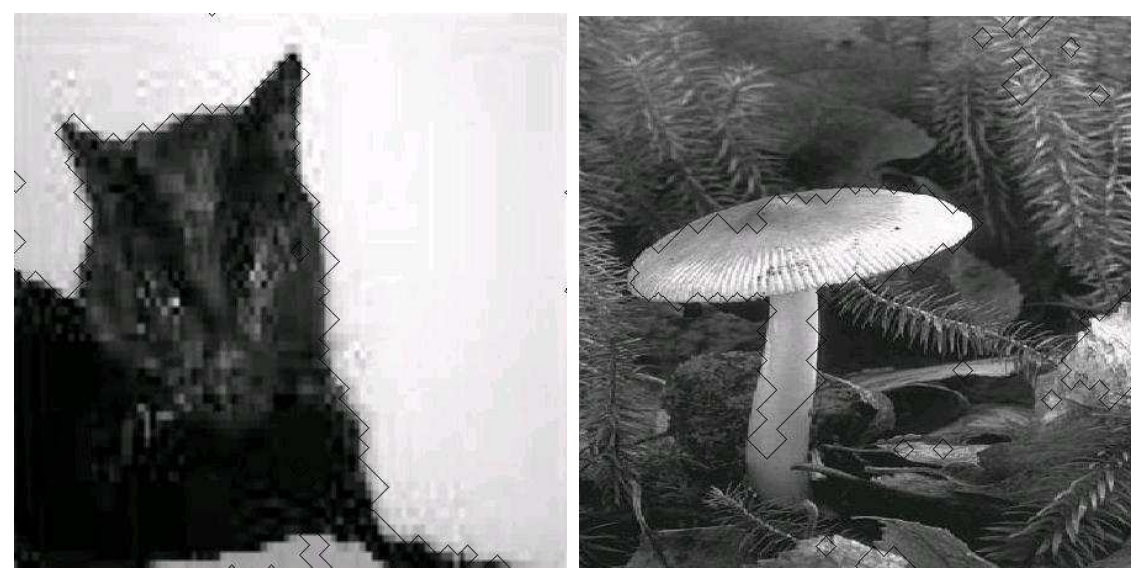

Figure 2: Segmented image of cat (left) and mushroom (right).

to evaluate our approach, as well as images from the PASCAL Network of Excellence challenge 2006

http://www . pascal-network. org/challenges/VOC/thumbs/VOC2006/index1.html

Figure 2 (left) depicts a cat. A target goodness-of-fit 0.07 was obtained after 134, 138 steps within 2 minutes. As for the toy examples, at each iteration, the disagreement loop dynamics were chosen with probability 0.7 , path creation and annihilation with probability 0.1 , and local polygon updates with probability 0.2 .

Figure 2 (right) shows a picture of a mushroom against a bushy background, with the segmentation result obtained after 150,000 steps. About 7.5 minutes computation time was needed to reach a 0.29 target goodness-of-fit. For this image, half of the update proposals involved local polygon modifications, the other half disagreement loops.

It should be emphasised that, unlike in the purely black-and-white set-up, for the grey level images the target goodness-of-fit (11) should not be identified with the misclassification rate because for instance pixels in the middle of the grey scale bring a contribution of 0.5 regardless of whether they are classified as black or white.

The discrete nature of polygonal fields defined using regular lattices implies that it is not possible in principle to follow exactly the outline of the foreground object. On the other hand, the state space of the annealing algorithm is much reduced, resulting in a faster procedure than that described for the continuum models by Kluszczyński et al. (2005, 2007). It should also be noted that we have used a simple $L_{p}$ criterion for 
optimisation; more sophisticated Hamiltonians would also include terms based on the local image gradient or shape and size characteristics of the polygonal contour configuration.

\section{Discussion and conclusion}

In this paper, we presented a class of Gibbs fields on finite graphs generated by regular tessellations, that can be interpreted as discretisations of the consistent polygonal Markov fields introduced by Arak \& Surgailis (1989). We showed that these fields enjoy a number of striking properties (including consistency, solvability, Markovianity) similar to those of the continuous polygonal Markov fields by which their definition was inspired. In particular, a dynamic representation can be constructed, which may be used to design simulation algorithms based on disagreement loops as well as path creation and annihilation. By addition of a Hamiltonian that measures the goodness-of-fit by an $L_{p}$ criterion, the model and techniques were applied to the image analysis problem of foreground-background separation.

It should be noted that, in contrast to the definition of a usual Markov random field, we have excluded $X$-shaped nodes, as doing so allows us to obtain a neat theory and avoids ambiguity in distinguishing the contours that comprise a polygonal configuration. The condition can easily be lifted, though, with only a few differences to the theory:

- In the dynamic construction developed in Section 3 we admit births of new particles also at sites where a prior collision occurred.

- The Hamiltonian $\Phi_{D}(\hat{\gamma})$ gets an extra term - $\log \left(1-\pi_{l_{1}} \pi_{l_{2}}\right)$ for each particle collision (death) site $n\left(l_{1}, l_{2}\right) \in D$ of $\gamma$ at which no new particle is born - this extra term takes into account the probability of not having an X-shaped node (not observing a new birth) at the death site $v\left(l_{1}, l_{2}\right)$, which is $1-\pi_{l_{1}} \pi_{l_{2}}$. Unfortunately, the so modified Hamiltonian becomes dependent on the choice of the coordinate system (spatial and time axes) unlike the original Hamiltonian in (5), this is because the notion of the birth site does depend on the direction of the time flow.

The algorithms proposed in this paper can be modified accordingly. Clearly, if a rich enough collection $\mathcal{T}$ is used, the choice to include or exclude $X$-shaped nodes hardly matters for segmentation purposes. 
Exploration of the full potential of the class of consistent polygonal fields built on finite tessellations is beyond the scope of the present paper and part of our work in progress. In particular, the choice of the collection $\mathcal{T}$ and the assignment of probabilities $\pi_{l}$ to each $l \in \mathcal{T}$ remains as subject of further study. From the perspective of image segmentation, it would be of particular interest to base the choice of tessellation on gradient information. Indeed, as edges lie at the boundaries between more or less homogeneous regions, they are characterised by abrupt changes in intensity values, and contain valuable information about the structures and objects present in the image. Hence, computation of the edge map of an image is useful, as the amount of data that needs to be stored may be greatly reduced, while preserving most of the information of interest (Rosenfeld \& Kak, 1982). The Canny (1986) filter is widely regarded as the best general purpose edge detector, designed to combine high signal-to-noise ratio and precise localisation and composed of Gaussian smoothing, Sobel filter, non-maximum suppression, and hysteresis steps.

We believe that foreground-background separation is not the only application for which the models and techniques discussed in this paper can be applied. As the Arak (1982) process can be extended to allow for more than two colour labels (Arak \& Surgailis (1991), Kluszczyński et al. (2007)), so is it of interest to explore their discrete counterparts for multi-class segmentation (current work in progress).

Another application area is the detection of linear features such as road networks or edges from image data. Several marked point process models have been proposed as prior distributions for this task (Lacoste (2004), Stoica (2001), Van Lieshout \& Stoica (2003)). However, being defined by a density with respect to a Poisson process, such line segment models are not capable to reproduce such characteristics of real-life images as an abundance of parallel lines, joined endpoints, or preferred angles of crossing. Consistent discrete polygonal field models, in contrast, are. Related, somewhat easier, conditional simulation problems occur in cognitive experiments aimed at understanding the human visual system.

Acknowledgements We gratefully acknowledge support from the EC 6th Framework Programme Priority 2 Information Society Technology Network of Excellence MUSCLE (Multimedia Understanding through Semantics, Computation and Learning; FP6-507752) which made possible mutual visits. Van Lieshout wishes to express gratitude for the warm hospitality and additional financial support received from the Nicolaus Copernicus University, Torun, Poland. The authors acknowledge R. Kluszczyński for technical assistance. 


\section{References}

Arak, T. (1982). On Markovian random fields with finite number of values. 4th USSRJapan symposium on probability theory and mathematical statistics, Abstracts of Communications, Tbilisi.

Arak, T., and Surgailis, D. (1989). Markov Fields with polygonal realisations. Probab. Theory Related Fields 80, 543-579.

Arak, T., and Surgailis, D. (1991). Consistent polygonal fields. Probab. Theory Related Fields 89, 319-346.

Arak, T., Clifford, P., and Surgailis, D. (1993). Point-based polygonal models for random graphs. Adv. in Appl. Probab. 25, 348-372.

Besag, J. (1986). On the statistical analysis of dirty pictures (with discussion). J. Roy. Statist. Soc. Ser. B 48, 259-302.

Canny, J. (1986). A computational approach to edge detection. IEEE Trans. PAMI 8, 679-698.

Chellapa, R., and Jain, A. (Eds.) (1993). Markov random fields: Theory and applications. Academic Press, Boston.

Clifford, P., and Middleton, R.D. (1989). Reconstruction of polygonal images. J. Appl. Stat. 16, 409-422.

Clifford, P., and Nicholls, G. (1994). A Metropolis sampler for polygonal image reconstruction. Available at:

http://www.stats.ox.ac.uk/ clifford/papers/met_poly.html

Geman, S., and Geman, D. (1984). Stochastic relaxation, Gibbs distributions and the Bayesian restoration of images. IEEE Trans. PAMI 6, 721-741.

Gimel'farb, G.L. (1999). Image textures and Gibbs random fields. Kluwer, Dordrecht.

Green, P.J. (1995). Reversible jump Markov chain Monte Carlo computation and Bayesian model determination. Biometrika 82, 711-732. 
Haario, H. and Saksman, E. (1991). Simulated annealing process in general state space. Adv. in Appl. Probab. 23, 886-893.

Møller, J., and Skare, Ø. (2001). Bayesian image analysis with coloured Voronoi tessellations and a view to applications in reservoir modelling. Statistical Modelling 1, 213-232.

Kluszczyński, R., Lieshout, M.N.M. van, and Schreiber, T. (2007). Image segmentation by polygonal Markov fields. To appear in Ann. Inst. Statist. Math.

Kluszczyński, R., Lieshout, M.N.M. van, and Schreiber, T. (2005). An algorithm for binary image segmentation using polygonal Markov fields. In: F. Roli and S. Vitulano (Eds.), Image Analysis and Processing, Proceedings of the 13th International Conference on Image Analysis and Processing. Lecture Notes in Comput. Sci. 3615, 383-390.

Lacoste, C. (2004). Extraction de réseaux linéiques à partir d'images satellitaires et aériennes par processus ponctuels marqués. PhD Thesis, University of Nice-Sophia Antipolis.

Lieshout, M.N.M. van, and Stoica, R.S. (2003). The Candy model: properties and inference. Statistica Neerlandica 57, 177-206.

Liggett, T. (1985). Interacting particle systems. Springer-Verlag, New York.

Nicholls, G.K. (1998). Bayesian image analysis with Markov chain Monte Carlo and coloured continuum triangulation models. J. R. Stat. Soc. Ser. B Stat. Methodol. 60, 643-659.

Rosenfeld, A., and Kak, A.C. (1982). Digital picture processing, volume II. Academic Press, New York, second edition.

Schreiber, T. (2005). Random dynamics and thermodynamic limits for polygonal Markov fields in the plane. Adv. in Appl. Probab. 37, 884-907.

Stoica, R. (2001). Processus ponctuels pour l'extraction des réseaux linéiques dans les images satellitaires et aériennes. PhD Thesis, University of Nice-Sophia Antipolis. 
Surgailis, D. (1991). The thermodynamic limit of polygonal models. Acta Appl. Math. 22, 77-102.

Winkler, G. (2003). Image analysis, random fields and Markov chain Monte Carlo methods, A mathematical introduction, 2nd ed. Applications of Mathematics, Stochastic Modelling and Applied Probability 27. Springer-Verlag, Berlin. 